\title{
The role of Arabidopsis ABA receptors from the PYR/PYL/RCAR family in stomatal acclimation and closure signal integration
}

\author{
Marcus Dittrich 1,2,12, Heike M. Mueller $\mathbb{B}^{3,12}$, Hubert Bauer ${ }^{3}$, Marta Peirats-Llobet $\mathbb{B}^{4,11}$, \\ Pedro L. Rodriguez $\mathbb{D}^{4}$, Christoph-Martin Geilfus $\mathbb{D}^{5}$, Sebastien Christian Carpentier ${ }^{6,7}$, \\ Khaled A. S. Al Rasheid ${ }^{8}$, Hannes Kollist ${ }^{9}$, Ebe Merilo ${ }^{9}$, Johannes Herrmann ${ }^{3}$, Tobias Müller ${ }^{1 \star}$, \\ Peter Ache $\mathbb{B}^{3}$, Alistair M. Hetherington ${ }^{10 \star}$ and Rainer Hedrich ${ }^{3}{ }^{3}$
}

\begin{abstract}
Stomata are microscopic pores found on the surfaces of leaves that act to control $\mathrm{CO}_{2}$ uptake and water loss. By integrating information derived from endogenous signals with cues from the surrounding environment, the guard cells, which surround the pore, 'set' the stomatal aperture to suit the prevailing conditions. Much research has concentrated on understanding the rapid intracellular changes that result in immediate changes to the stomatal aperture. In this study, we look instead at how stomata acclimate to longer timescale variations in their environment. We show that the closure-inducing signals abscisic acid (ABA), increased $\mathrm{CO}_{2}$, decreased relative air humidity and darkness each access a unique gene network made up of clusters (or modules) of common cellular processes. However, within these networks some gene clusters are shared amongst all four stimuli. All stimuli modulate the expression of members of the PYR/PYL/RCAR family of ABA receptors. However, they are modulated differentially in a stimulus-specific manner. Of the six members of the PYR/PYL/RCAR family expressed in guard cells, PYL2 is sufficient for guard cell ABA-induced responses, whereas in the responses to $\mathrm{CO}_{2 \prime}$ PYL4 and PYL5 are essential. Overall, our work shows the importance of $A B A$ as a central regulator and integrator of long-term changes in stomatal behaviour, including sensitivity, elicited by external signals. Understanding this architecture may aid in breeding crops with improved water and nutrient efficiency.
\end{abstract}

S tomata are pores on the leaf surface that are bounded by two guard cells. Their role is to optimize gas exchange in changing environmental conditions ${ }^{1-3}$. In the light, stomata open, allowing $\mathrm{CO}_{2}$ to enter the leaf for photosynthesis. In contrast, darkness, high concentrations of $\mathrm{CO}_{2}$, reductions in relative air humidity $(\mathrm{RH})$ that are equivalent to an increase in the water vapour pressure deficit (VPD) and exposure to the hormone abscisic acid (ABA) all promote stomatal closure ${ }^{4-8}$. This is a rapid process typically lasting from 20 to $40 \mathrm{~min}$. Reductions in stomatal aperture are the result of turgor and volume reduction in the respective guard cell pair. The key player in $\mathrm{ABA}$ - and $\mathrm{CO}_{2}$-dependent guard cell deflation is the guard cell anion channel SLAC1, together with the SLAC1activating protein kinase, OST1 (ref. ${ }^{9}$ ). Under low, subthreshold concentrations of ABA, the protein phosphatase ABI1 keeps the guard cell anion channel kinases dephosphorylated and SLAC1 inactive $^{10}$. When the concentration of ABA increases it binds to receptors of the PYL/PYR/RCAR family ${ }^{11-13}$. ABA receptor interaction prevents the ABI1-mediated inactivation of the SLAC1 kinase. This results in anion channel opening, which in turn brings about depolarization of the plasma membrane. The change in membrane potential results in the release of osmotically active $\mathrm{K}^{+}$and anions. This is followed by osmotic water release causing guard cell deflation and stomatal closure $e^{4,9,14,15}$.

Over the last 40 years most investigations have concentrated on identifying and understanding how individual guard cell proteins and intracellular second messengers interact to bring about these rapid changes in stomatal aperture. In contrast, we know much less about the processes that underlie stomatal acclimation to longer term environmental changes ${ }^{16}$. These involve alterations in gene expression, which result in changes in stomatal development and function. In the former category it is recognized that plants acclimate to increases in the concentration of atmospheric carbon dioxide and light intensity by decreasing the number or size of stomata that develop on the surface of the plant leaves ${ }^{17-21}$. Reducing stomatal density is known to increase plant water-use efficiency ${ }^{22}$. Stomata also acclimate to changing environments by altering their sensitivity to ABA. In Arabidopsis this happens during development, with the youngest guard cells being ABA insensitive $^{23}$. Guard cell sensitivity to ABA is modulated by water stress or exposure to increased $\mathrm{VPD}^{23,24}$. In these cases, 'switching on'

'Department of Bioinformatics, University of Würzburg, Würzburg, Germany. ${ }^{2}$ Department of Human Genetics, University of Würzburg, Würzburg, Germany. ${ }^{3}$ nstitute for Molecular Plant Physiology and Biophysics, Biocenter, University Würzburg, Würzburg, Germany. ${ }^{4}$ Instituto de Biología Molecular y Celular de Plantas, Consejo Superior de Investigaciones Cientificas-Universidad Politecnica de Valencia, Valencia, Spain. ${ }^{5}$ Division of Controlled Environment Horticulture, Faculty of Life Sciences, Albrecht Daniel Thaer-Institute of Agricultural and Horticultural Sciences, Humboldt-University of Berlin, Berlin, Germany. ${ }^{6}$ YYBIOMA, Proteomics Core Facility, KU Leuven, Leuven, Belgium. ${ }^{7}$ Division of Crop Biotechnics, Department of Biosystems, KU Leuven, Leuven, Belgium. ${ }^{8}$ Zoology Department, College of Science, King Saud University, Riyadh, Saudi Arabia. ${ }^{9}$ Institute of Technology, University of Tartu, Tartu, Estonia. ${ }^{10} \mathrm{~S} c h o o l$ of Biological Sciences, University of Bristol, Bristol, UK. "1Present address: Centre for AgriBioscience, Department of Animal, Plant and Soil Science, School of Life Science, La Trobe University, Bundoora, Victoria, Australia. ${ }^{12}$ These authors contributed equally: Marcus Dittrich, Heike M. Mueller. *e-mail: Tobias.Mueller@biozentrum.uni-wuerzburg.de; Alistair.Hetherington@bristol.ac.uk 
ABA responsiveness provides the plant with the ability to control its water relations.

Understanding how stomata acclimate to changes in their environment has clear relevance to the global challenge of producing crops that are more resilient to changes in the environment. Here we identify changes in guard cell gene expression that are likely to contribute to the mechanisms that allow stomatal aperture responses to adapt in the longer term to changes in closure-inducing environmental signals. We reveal that these responses are characterized by the expression of both stimulus-specific suites of genes and core genetic modules that are regulated by all closure-inducing stimuli. We also suggest that part of stomatal acclimation to all these signals is achieved by controlling the sensitivity of the guard cell to ABA. This is achieved by differentially regulating the expressions of members of the PYR/PYL/RCAR family of ABA receptors. Moreover, we show, in the cases of $\mathrm{ABA}$ and elevated $\mathrm{CO}_{2}$, that response specificity is achieved by these signals accessing different members of the PYR/PYL/RCAR family of receptors.

Stimulus-specific modulation of guard cell gene expression. To investigate the effects of ABA, low air humidity/increased VPD, darkness and elevated $\mathrm{CO}_{2}$ on guard cell gene expression, we used a microarray-based approach. We reanalysed two microarray datasets (ABA and RH/VPD treatment) from a previous study ${ }^{6}$ and combined them with two new microarray studies where we used darkness and high $\mathrm{CO}_{2}$ as stomatal closing signals (for detailed analysis of the differentially expressed genes see Supplementary Table 1a). We first analysed the resulting dataset using classical pathway analyses and this revealed that each closure-inducing signal addressed a defined, unique cluster of gene pathways (Fig. 1, Supplementary Fig. 1a and Supplementary Information 1). We also found that gene clusters are shared among two, three or all four stimuli. For example, the shared clusters for ABA and lowered $\mathrm{RH}$ (equivalent to increased VPD) are dominated by the ABA response (Supplementary Fig. 1b) and the late embryogenesis abundant pathways and are upregulated by both treatments. To gain further information about the interrelationship of these pathways we analysed the coexpression patterns of the genes from each pathway using the public ATTED II database ${ }^{25}$. This analysis revealed that both pathways are highly interconnected (Supplementary Fig. 1c-e). Notably, RAB18, DAA1 and MYB74, which we had previously identified as being core to RH- (VPD) and ABA-induced guard cell responses ${ }^{26}$, were coexpressed with several other genes common to both responses, such as the dehydrins and members of the HVA22 family (Supplementary Fig. 1c-e).

The shared gene cluster of darkness and low RH (high VPD) is dominated by abiotic stress and glucosinolate pathway genes. Both clusters contain flavin monooxygenases/glucosinolate oxygenases (FMOGS), which are responsible for the last step of side-chain modification of aliphatic glucosinolates. FMOGS are involved in biotic and abiotic stress responses in general and most of them are induced by $\mathrm{ABA}^{27}$. The shared clusters for darkness/CO, $\mathrm{ABA} /$ darkness, $\mathrm{ABA} /$ $\mathrm{CO}_{2}$ and $\mathrm{RH} / \mathrm{CO}_{2}$ are dominated by more general metabolic pathways, receptor kinases, cell wall degradation, major carbohydrate metabolism, glucosidases and transport (Supplementary Fig. 1a). To directly model these complex interconnections we performed an integrative network analysis on the basis of a rigorous mathematical approach (see Methods).

Specifically, we incorporated the set of protein-protein interactions from the STRING database, which primarily represents physical interactions between proteins, into our analysis. By integrating these data we added a new dimension into the expression data. In contrast to the coexpression network, this allows the detection of functional signalling links at the posttranslational protein level. We analysed the network on the basis of the measured expression levels into functionally coherent modules. These can be regarded as the building blocks of cellular processes, such as basic signalling cascades. The resulting

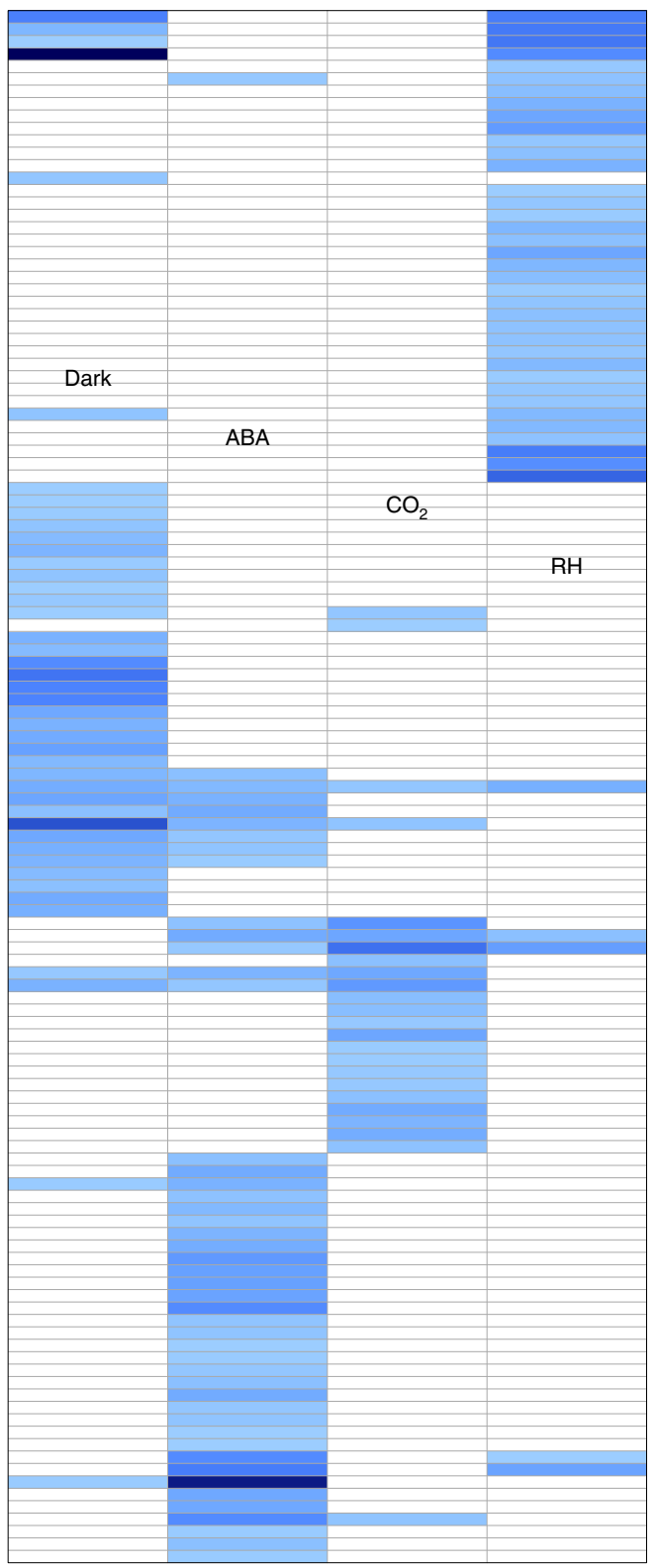

Fig. 1 | Guard cell signalling pathway analysis. MapMan pathways with a significant enrichment in at least one stimulus are visualized in a heat map. Pathways are listed in rows and stimuli in columns. Darker shades (corresponding to the negative decadic logarithm of the enrichment $P$ value) depict more significant enrichments. For each treatment and the respective controls, four microarrays were analysed, except darkness $(n=3)$. All replicates represent biologically independent samples. A competitive pathway enrichment test was performed as implemented in the camera function from the limma package based on the gene-expression data.

optimal solutions represent maximally responsive network modules with respect to the specific experimental stimuli.

This approach is hypothesis-free and allows an unbiased exploration of stimulus-triggered network responses, and, in particular, can integrate novel, hitherto unknown, genes and connections into a pathway. Based on this algorithm we were able to expose clusters and connections that cannot be easily detected by classical methods. This revealed stimulus-specific gene modules responsive to different stomata-closing signals (Fig. 2a-d) (for details see Supplementary Information 2, Supplementary Table $1 \mathrm{~b}$ and Supplementary Fig. 2a-d). 

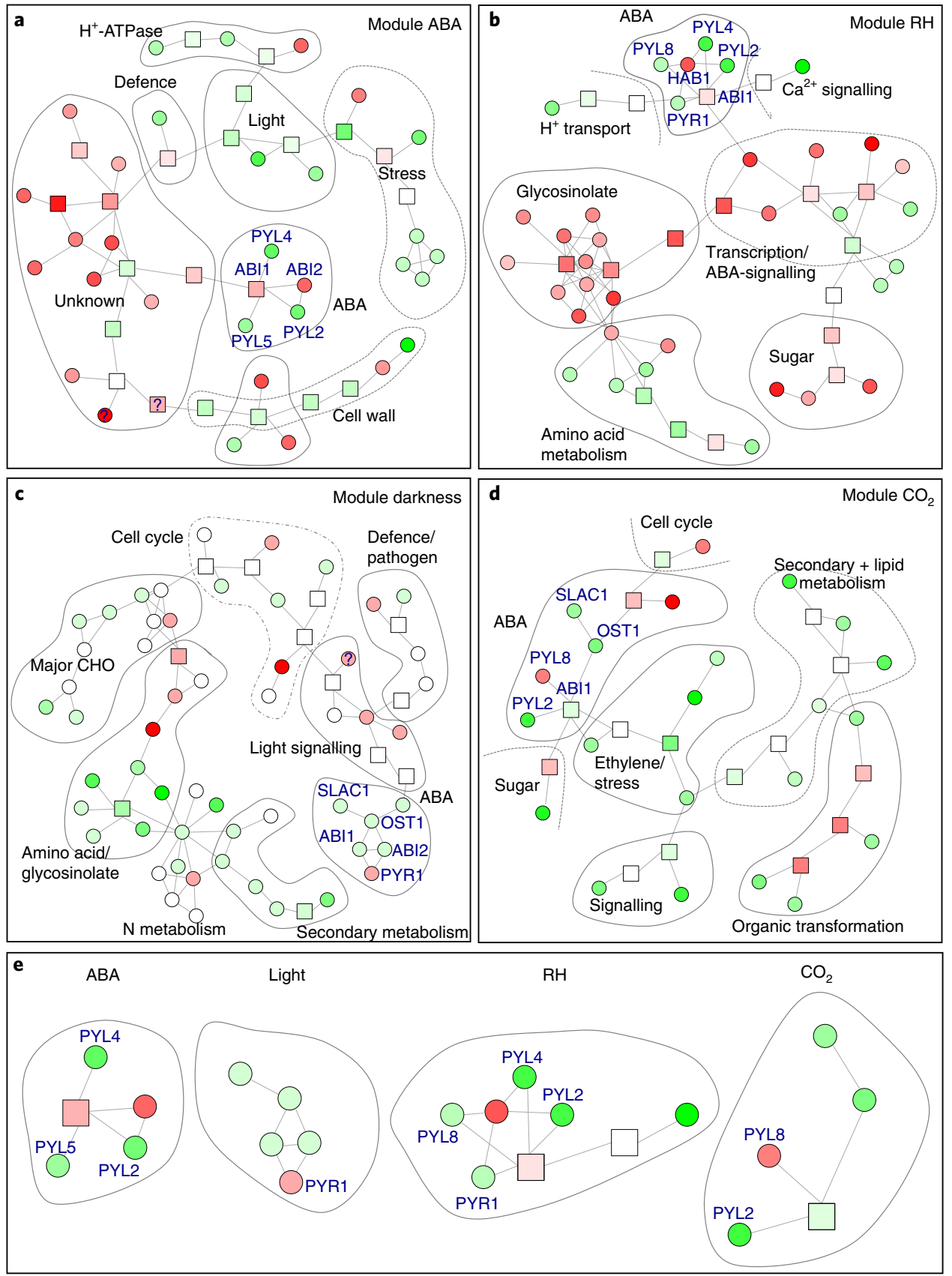

Fig. 2 | Integrative networks analysis identifies distinct stomatal closing signal modules. a-d, Stimulus-specific modules: optimal responsive subnetworks of regulated genes in the entire network for $\mathrm{ABA}(\mathbf{a}), \mathrm{RH}(\mathbf{b})$, darkness (c) and $\mathrm{CO}_{2}(\mathbf{d})$ stimulation. Circles denote positive-scoring genes (with highly significant expression changes), whereas rectangles denote negative-scoring nodes (for example, additional nodes of modest regulation), which are implicated by the algorithm on the basis of their functional network context. Red indicates upregulation and green represents downregulation, where darker colours correspond to higher fold change values. Gene clusters of common cellular processes are enclosed by shaded lines highlighting the functional substructure of the modules. Only ABA-responsive genes are named (for details see Supplementary Fig. 2). e, ABA receptors are the main components in adaptation to stomatal closing signals. Each module contains a cluster of typical ABA-responsive genes. In all cases, $A B A$ receptors are affected. Among the closing signals, the kind of receptors as well as the direction of regulation differ markedly, indicating an altered sensitivity towards $A B A$ in the adaptation process to the individual signals. Blue text indicates genes known to be involved in ABA signalling. For each treatment, and the respective controls, four microarrays were analysed, except darkness $(n=3)$. All replicates represent biologically independent samples. The network analysis is based on the statistics and $P$ values described in Supplementary Table 1 a.

Closure-inducing signals differentially modulate PYR/PYL/ RCAR receptor expression. Another feature to emerge from our investigations was that the different closure-inducing signals led to differential expression of ABA receptor family members in guard cells. At the level of the whole plant, and at the tissue level, it has been documented that $\mathrm{ABA}$ application regulates the expression of certain PYR/PYL/RCAR receptors ${ }^{13,28}$. In our investigations we found that six guard cell-localized $P Y R / P Y L / R C A R s$ were affected (Fig. 2e). In the case of ABA, three receptors (PYL2, PYL4 and PYL5) were downregulated, while darkness upregulated PYR1. 
Exposure to dry air (increased VPD) resulted in the downregulation of PYR1, PYL2, PYL4 and PYL8. In high $\mathrm{CO}_{2}$ PYL8 is upregulated while PYL2 is downregulated. These data suggest that ABA and receptors of the $P Y R / P Y L / R C A R$ family are involved in the response to all these closure-inducing stimuli. One possible interpretation of these results is that, in the long term, acclimation to these signals is achieved through modulating the transcript abundance of individual $P Y R / P Y L / R C A R$ family members. The net result of this would be to fine-tune guard cell sensitivity to ABA. In this context it is interesting that we found that the transcript abundance for these receptors was differentially sensitive to ABA concentration. Specifically, we observed that $250 \mathrm{nM}$ ABA induced a reduction in PYL4 abundance, while changes in the abundance of PYL2, PYL4, PYL5, PYL8 and PYR1 were only observed on treatment with ten times this concentration of ABA $(2.5 \mu \mathrm{M})$. In this context, it should be mentioned that the products of ABA metabolism might interact with ABA receptors. This is indeed the case for phaseic acid, which is capable of binding to PYL2 (ref. ${ }^{29}$ ). It is therefore possible that these metabolic products could also regulate transcriptional changes of the receptor genes. We next investigated whether other guard cell ABA-regulated genes were, like the $P Y R / P Y L$ genes, differentially regulated in a concentration-dependent manner. As is apparent from the data in Table 1 and Supplementary Fig. 3 (see Supplementary Fig. 3 and Methods for details and statistics), ABR, HAI1 and MYB60 responded to ABA stimulation in a concentration-dependent manner. Together, these observations suggest a framework in which $\mathrm{ABA}$ regulates specific receptor family member abundance in a concentration-dependent manner. This would potentially provide a high degree of control over receptor and response sensitivity.

Differential guard cell gene expression regulated by PYR/PYL family members. We next decided to investigate the possibility that different members of the PYR/PYL/RCAR receptor family differentially regulate gene expression. For this we used Arabidopsis PYR/ $P Y L / R C A R$ receptor mutants. The Arabidopsis genome contains 14 $P Y R / P Y L / R C A R$ genes and previous studies have shown that generation of quadruple, pentuple and sextuple mutants is required to obtain robust ABA-insensitive phenotypes ${ }^{12,28,30}$. However, both biochemical analyses of different receptor-phosphatase complexes and of receptor gene-expression patterns suggest that the function of $\mathrm{ABA}$ receptors is not completely redundant ${ }^{28,31,32}$. Analysis of the single pyl8 mutant, for example, has revealed a non-redundant role of PYL8 in root sensitivity to $\mathrm{ABA}^{33,34}$, while the PYL9/RCAR1 receptor specifically regulates the protein phosphatase AHG1 (ref. ${ }^{32}$ ) and promotes leaf senescence ${ }^{35}$. Additionally, a non-redundant function of the dimeric receptor BdPYL1 has been reported in Brachypodium $^{36}$. Functional diversification follows the evolutionary expansion of a gene family and, therefore, we investigated the role of different ABA receptors in guard cells.

Our transcriptomic data confirmed that guard cells express the six ABA receptors PYR1, PYL1, PYL2, PYL4, PYL5 and PYL8 that have previously been shown to be relevant for stomatal closure ${ }^{7,28}$. We therefore asked whether, in guard cell ABA signalling, the six different receptors are redundant, or if each $P Y R / P Y L$ has individual functions. To answer this question, we used the $p y r / p y l$ quintuple mutant known as 12458 (ref. ${ }^{28}$ ), where the receptors PYR1 and PYL2, PYL4, PYL5 and PYL8 are knocked out, and the 11458 (ref. ${ }^{33}$ ) loss-of-function mutant in which PYR1 and PYL1, PYL4, PYL5 and PYL8 are knocked out. In addition, we took advantage of the availability of quintuple mutant complementation lines. These lines, under the control of the guard cell-specific promoter GC1 (At1g22690) ${ }^{37}$, express single receptors individually (see details in Supplementary Fig. 4).

To exclude the possibility that the following results were influenced by overexpression of the individual receptors in the
Table 1 | ABA dose-response of the transcription of guard cell ABA-receptor and ABA-regulated genes

\begin{tabular}{|c|c|c|c|c|c|c|}
\hline \multirow[t]{2}{*}{ Gene } & \multirow[t]{2}{*}{ Locus } & \multicolumn{5}{|c|}{ ABA concentration } \\
\hline & & $25 n M$ & $100 \mathrm{nM}$ & $250 \mathrm{nM}$ & $2.5 \mu \mathrm{M}$ & $25 \mu \mathrm{M}$ \\
\hline PYR1 & At4g17870 & & & & & $64^{a}$ \\
\hline PYL1 & At5g46790 & & & & & \\
\hline PYL2 & At2g26040 & & & & $39^{a}$ & $18^{\mathrm{a}}$ \\
\hline PYL4 & At2g38310 & & & $66^{a}$ & $36^{a}$ & $19^{a}$ \\
\hline PYL5 & At5g05440 & & & & $33^{a}$ & $16^{a}$ \\
\hline PYL8 & At5g53160 & & & & $77^{a}$ & $54^{a}$ \\
\hline MYB60 & At1g08810 & & & & & $42^{a}$ \\
\hline$A B R$ & At3g02480 & & & & $1,331^{b}$ & $5,679^{b}$ \\
\hline HAl1 & At5g59220 & & $173^{b, c}$ & & $594^{b}$ & $1,535^{b}$ \\
\hline
\end{tabular}

On ABA spray application, transcription was either induced, reduced or remained unchanged. Numbers represent the expression changes presented in Supplementary Fig. 3 (in percentages) relative to untreated control. Blank entry, no significant change in expression; adownregulation; ${ }^{b}$ upregulation; ' denotes that the value has weak significance and represents only $11 \%$ of the maximum induction (for sample sizes and statistics, see related Supplementary Fig. 3 and Methods).

complementation lines, we measured the expression levels of PYR1, PYL1, PYL2, PYL4, PYL5 and PYL8 in guard cells of wild type, both quintuple mutants and the complementation lines in the 12458 background (Supplementary Fig. 5a-f). These results showed that neither PYL1 (present in 12458) nor PYL2 (present in 11458) were upregulated in the quintuple mutants to compensate for the lack of the ABA receptors that were knocked out. Furthermore, the complementation lines showed wild type-like expression of the respective receptors (maximally varying about 2-3-fold). To confirm that ABA receptor RNA abundance is mirrored at the protein level, we selected PYL2 as a test case for a mass spectrometry-based proteomic approach, because PYL2 is the most relevant for ABA-induced stomatal closure (see 'Loss of PYL2 gene renders stomata ABA insensitive'). As expected PYL2 receptor peptides were neither found to be present in the 12458 mutant, nor in the associated PYR1, PYL4 and PYL5 complementation lines. In wild-type guard cells and those of the PYL2complemented 12458 mutant, however, PYL2 peptides were detected at comparable levels (Supplementary Fig. 5g). These findings underline the suitability of the GC1 promoter for driving almost physiological guard cell ABA receptor expression.

To resolve whether different members of the PYL/PYR receptor family regulated expression of guard cell genes in a differential fashion we concentrated on the ability of ABA to regulate the following known guard cell ABA-responsive genes: MYB60, ABI1, ABI2, CIPK25 and KCR2 (ref. ${ }^{6}$ ) (see Table 2 and Supplementary Fig. 6 for details and statistics). We observed MYB60 downregulation in wild type and the 11458 (PYL2 present) mutant, but not in the 12458 (PYL1 present) mutant. Complementation of the latter mutant with PYR1 or PYL2 restored the wild-type phenotype, indicating that ABA-dependent downregulation of MYB60 is under the control of these receptors. Upregulation of $\mathrm{ABI} 2$ expression was abolished in 11458 but it was restored by PYR1 or PYL1 complementation, indicating that PYR1 and PYL1 are sufficient for ABI2 expression. Likewise, upregulation of CIPK25 was abolished in 11458 but restored by PYL1 or PYL8, indicating that they are sufficient for CIPK25 expression. Complex regulation of KCR2 expression seems to occur, since ABA-induced upregulation was abolished in 11458 (PYL2 present) and could not be restored in complementation lines. Finally, although RAB18 appeared to be induced by ABA always, the presence of PYL1 in the 12458 mutant $(P=0.00018)$ or combined with PYR1 $(P=0.025)$ led to a 12 - to 14 -fold induction (more 


\begin{tabular}{|c|c|c|c|c|c|}
\hline & MYB60 & $\mathrm{ABI} 2$ & CIPK25 & KCR2 & RAB18 \\
\hline Wild type & $26^{a}$ & $268^{b}$ & $268^{b}$ & $195^{b}$ & $400^{b}$ \\
\hline $\begin{array}{l}12458 \text { (PYL1 } \\
\text { present) }\end{array}$ & & $298^{b}$ & $269^{b}$ & $285^{b}$ & $1,218^{b}$ \\
\hline PYR1::12458 & $40^{\mathrm{a}}$ & & $303^{b}$ & $194^{b}$ & $1,436^{b}$ \\
\hline PYL2::12458 & $38^{a}$ & $251^{b}$ & $228^{b}$ & $200^{b}$ & $731^{b}$ \\
\hline PYL4::12458 & & $307^{b}$ & $331^{b}$ & $244^{b}$ & $491^{b}$ \\
\hline PYL5::12458 & & $314^{b}$ & & & $381^{b}$ \\
\hline $\begin{array}{l}11458 \text { (PYL2 } \\
\text { present) }\end{array}$ & $31^{a}$ & & & & $649 b$ \\
\hline PYR1::11458 & $22^{\mathrm{a}}$ & $203^{b}$ & & & $956^{b}$ \\
\hline PYL1::11458 & $14^{a}$ & $160^{b}$ & $178^{b}$ & & $433^{b}$ \\
\hline PYL4::11458 & $25^{\mathrm{a}}$ & & & & $421^{b}$ \\
\hline PYL5::11458 & $25^{a}$ & & & & $301^{b}$ \\
\hline PYL8::11458 & $43^{a}$ & & $215^{b}$ & & $493^{b}$ \\
\hline \multicolumn{6}{|c|}{$\begin{array}{l}\text { ABA-spraying }(25 \mu \mathrm{M}) \text { led to up- or downregulation of genes in wild-type plants. Quintuple } \\
\text { knockout and complementation lines revealed the receptors necessary for ABA-induced gene } \\
\text { regulation. Numbers represent the expression changes presented in Supplementary Fig. } 6 \\
\text { (in percentages) relative to untreated control. 'Downregulation; bupregulation; blank cell, no } \\
\text { significant change in expression (for sample sizes and statistics see related Supplementary Fig. } 6 \\
\text { and Methods). }\end{array}$} \\
\hline
\end{tabular}

than three times that of the wild type), but, when just one of the receptors PYL2, PYL4 or PYL5 was expressed, this overinduction was suppressed. Since we found no strong differences in the basal levels of the examined genes in the different genetic backgrounds (Supplementary Table 1c), these findings indicate that transcriptional guard cell ABA responses are mediated by individual receptors or distinct $P Y R / P Y L$ combinations.

Loss of PYL2 gene renders stomata ABA insensitive. The above experiments reveal the role of individual PYR/PYL family ABA receptors in the control of guard cell gene expression. We next turned our attention to investigating whether individual receptor family members have distinct roles to play in the control of stomatal aperture and gas exchange. To do this we first used infrared gas analysis (IRGA) ${ }^{38}$. In this approach we used excised leaves and applied ABA via the petioles to exclude any effect of the roots on transpiration. Under these conditions wild-type leaves opened their stomata on illumination and adjusted their aperture to the environmental settings before application of $\mathrm{ABA}^{39}$. We found that, in the light, the 12458 quintuple mutant (PYL1 present), in contrast to the 11458 mutant (PYL2 present), exhibited a severe open-stomata phenotype that manifested itself in rapid wilting on exposure to light. To overcome this experimental difficulty, we preincubated plants in the dark with $25 \mu \mathrm{M}$ ABA for $1 \mathrm{~h}$ before applying the light stimulus. Using this protocol, on illumination, wild-type plants remained in a non-transpiring state (that is, ABA inhibited lightinduced stomatal opening). In contrast, transpirational water loss of the ABA-insensitive 12458 mutant increased over time (Fig. 3a), whereas the 11458 quintuple mutant, which expressed wild-type PYL2, behaved almost like wild type (Fig. 3a). Thus, we tested all available complementation lines in the 12458 background. The 12458 mutant, when complemented by PYR1, exhibited reduced transpiration, but the presence of PYR1 did not fully complement the ABA-insensitive phenotype of the quintuple mutant that had lost PYL2 (Fig. 3b). These data suggest a key role for PYL2 in the guard cell ABA-signalling pathway that leads to stomatal closure. This was confirmed by complementing PYL2 in the 12458 mutant, which resulted in a plant that showed the same ABA suppression of
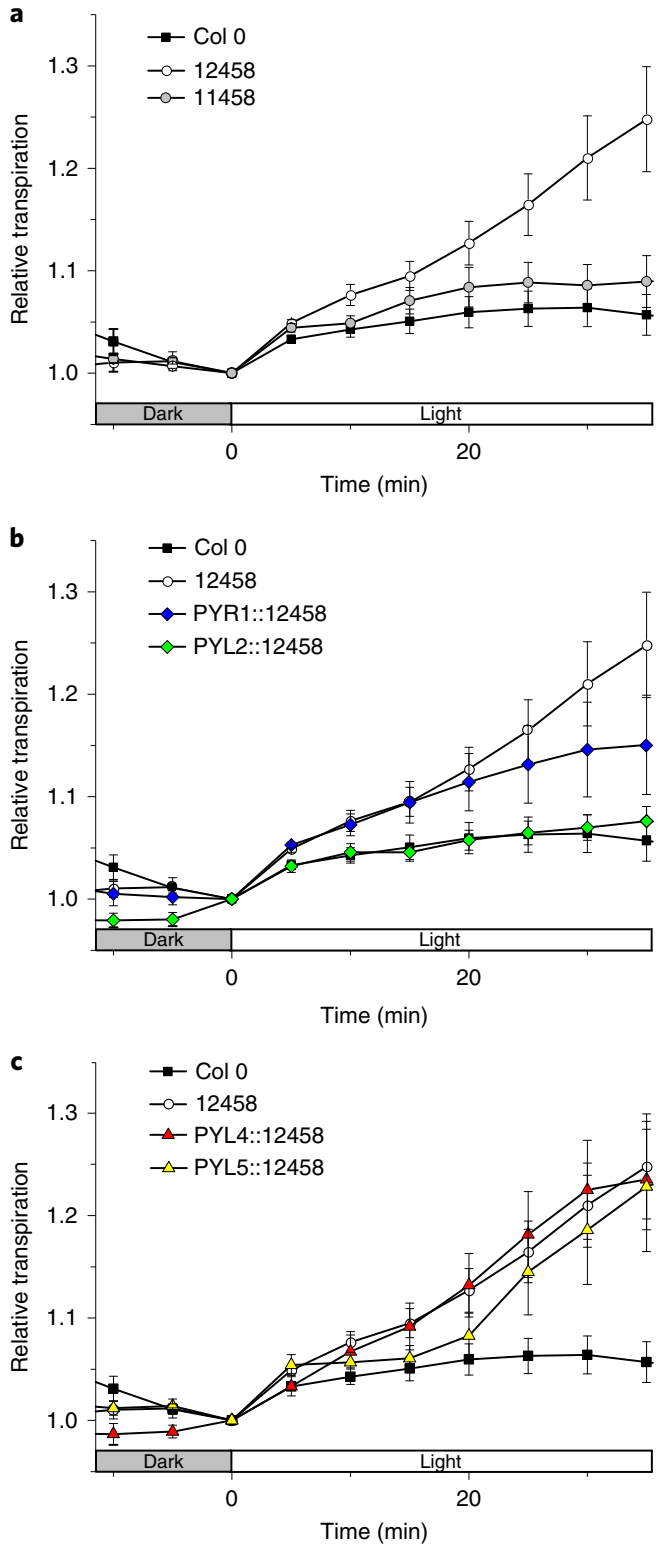

Fig. 3 | PYL2 is sufficient for stomatal ABA sensitivity. Excised leaves, in darkness, were preincubated with $A B A$ fed via the petiole to prevent stomatal opening in the light. The stomatal aperture was measured by gas exchange as a function of relative transpiration. a, Stomata of wild type and the 11458 mutant (PYL2 present) remained almost closed, while those of the 12458 mutant appeared to be ABA insensitive. b, PYL2 in the 12458 background fully restored the wild-type phenotype, while PYR1 reacted with incomplete ABA sensitivity. c, PYL4 and PYL5 were unable to restore the ABA sensitivity of the 12458 quintuple background line. Values are normalized to time point $0 . n=28$ for Col $0, n=6$ for $12458, n=9$ for 11458 , $n=5$ for PYR1::12458, $n=5$ for PYL2::12458, $n=6$ for PYL4::12458, $n=7$ for PYL5::12458. All replicate numbers represent biologically independent samples, mean \pm s.e.m.

stomatal transpiration as the wild type (Fig. 3b). In contrast, guard cell expression of PYL4 and PYL5 did not complement the rapidly wilting 12458 mutant phenotype (Fig. 3c).

In addition to IRGA measurements, which in this case provide data on the effects of manipulating gene expression on stomatal transpirational control at the level of the whole leaf, we followed the response of individual stomata in their natural environment of 
intact leaves. This was achieved by applying $50 \mu \mathrm{M}( \pm)$-ABA (corresponding to $25 \mu \mathrm{M}$ biologically active $\mathrm{ABA}$ ) to single stoma via micropipette-assisted electro-infusion. By introducing ABA directly to the guard cells we aimed to exclude any indirect hormone effects, via, for example, the mesophyll cells and/or vasculature, on the control of transpiration by stomata. Using this protocol we observed that stomata of wild-type Arabidopsis closed within $10 \mathrm{~min}$ of exposure to ABA (Fig. 4a,b and Supplementary Videos 1 and 2). In contrast, stomata of the 12458 mutant (PYL1 present) did not respond to the hormone and remained open. This mutant was also the only one with substantially wider open stomata under control conditions (Fig. 4c). In the quintuple mutant (12458) complemented by PYL2 and the 11458 mutant (PYL2 present), we observed stomatal closure that was identical to wild type. We also observed that PYL5 was not able to complement the ABA-insensitive phenotype (Fig. 4a,b and Supplementary Videos 1 and 2). This demonstrates that PYL2 is sufficient to fully rescue the guard cell ABA signalling and fast stomatal closure. To confirm our findings that PYL2 and, partially, PYR1 are able to complement the ABA insensitivity of the 12458 mutant, we repeated the $A B A$ preincubation experiment with the $p y l 2-1$ loss-offunction mutant (seed strain GT2864) ${ }^{12}$ and the corresponding Ler wild type strain. Although PYR1, PYL1, PYL4, PYL5 and PYL8 are present in this mutant, pyl2-1 behaved almost like the PYR1 complementation line in the 12458 background (Supplementary Fig. 7), supporting our hypothesis that PYL2 and PYR1 are the key receptors for proper ABA-induced stomatal closure.

PYL4 and PYL5 are sufficient for $\mathrm{CO}_{2}$-induced stomatal closure. Since Raschke's research in the 1970s we have known that stomata require $\mathrm{ABA}$ to sense elevated $\mathrm{CO}_{2}$ (refs. ${ }^{7,8,40,41}$ ). However, the molecular basis for this interaction has not been clear. We took advantage of the panel of ABA receptor mutants previously described to investigate the basis of this interaction using IRGA. Stomata that had been opened in the light in the presence of $400 \mathrm{ppm}$ $\mathrm{CO}_{2}$ (ambient) were exposed to $1,000 \mathrm{ppm} \mathrm{CO}_{2}$. As expected, this resulted in a decrease in transpiration consistent with a reduction in stomatal aperture (Fig. 5a). Next, we used the 11458 mutant, in which PYL2 is expressed naturally. We had previously shown that this PYL2-expressing mutant showed a wild type-like ABAinduced closure response (Fig. 3b). However, in contrast to ABA, $\mathrm{CO}_{2}$-induced closure was impaired in this mutant, as well as in the 12458 mutant (Fig. 5a). Stomata of complementation lines in the 12458 background (with PYL1 present) expressing PYR1 or PYL2 also remained impaired in their response to high $\mathrm{CO}_{2}$ (Fig. 5b). However, lines that express PYL4 or PYL5 in guard cells regained stomatal $\mathrm{CO}_{2}$ sensitivity that was similar to wild-type plants (Fig. 5c). These data show that PYL4 or PYL5 are sufficient for the induction of closure by increased $\mathrm{CO}_{2}$. Interestingly, except for the PYL5 complementation line, all other mutants showed remarkably higher ground levels of transpiration in the light and under ambient $\mathrm{CO}_{2}$ atmosphere compared with wild type (Supplementary Fig. 8). Only the PYL4 complementation line started to reopen their stomata after about $2 \mathrm{~h}$ of high $\mathrm{CO}_{2}$ treatment. Future studies will investigate whether this behaviour represents a phenomenon associated with adaptation (Supplementary Fig. 8).

\section{Discussion}

The primary objective of the work described in this paper has been to explore how stomata adapt to changed environmental conditions. Our focus has not been on changes in stomatal development that are induced by changes in the environment. Rather, we have concentrated on examining the mechanisms likely to underpin changes in, for example, the sensitivity of the stomatal aperture response to closure-inducing stimuli. Specifically, when we looked at four different closure-inducing stimuli, we found that they regulated a diverse set of intracellular pathways. While there was some overlap, a

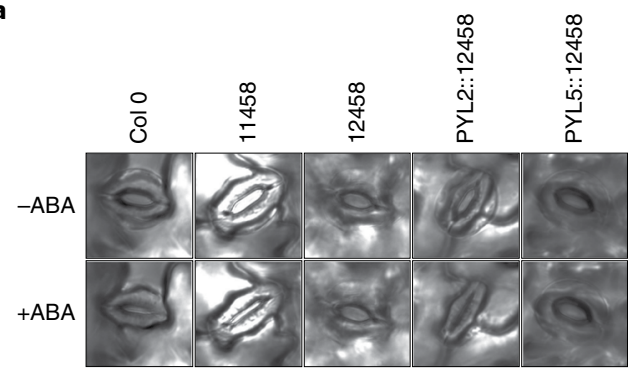

b
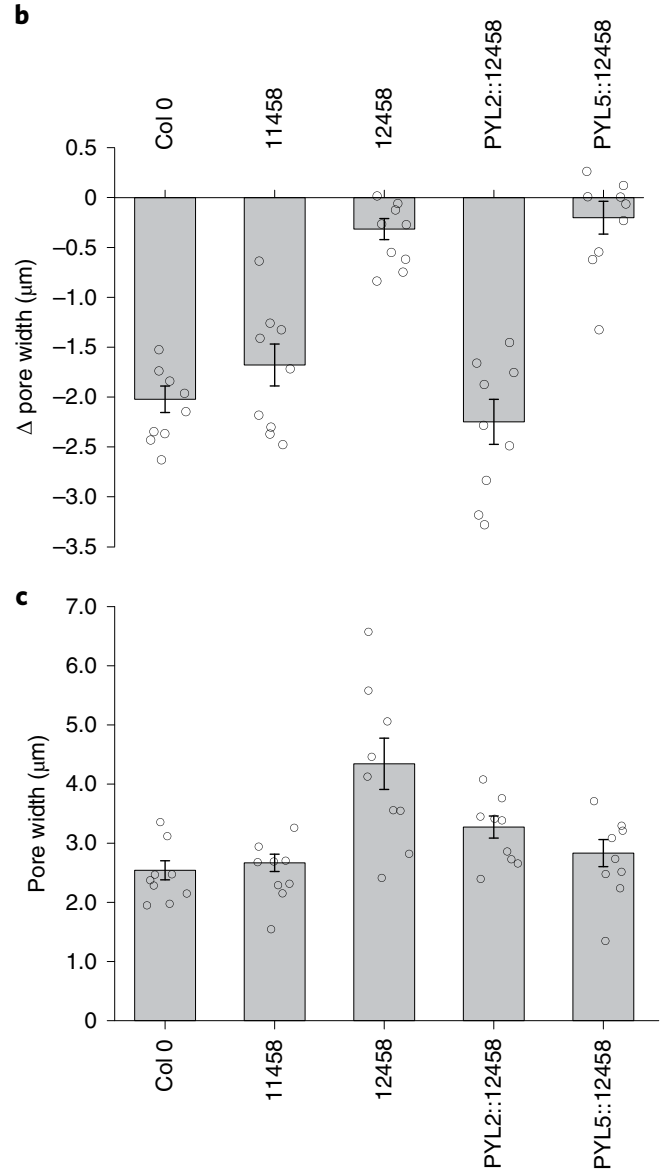

Fig. 4 | $A B A$-induced movements of individual stoma. Electro-infusion of ABA to single stoma. a, Stomata of wild type, 11458 mutants (PYL2 present) and the PYL2::12458 complementation line closed within $10 \mathrm{~min}$ following an ABA pulse. In contrast, stomata of the 12458 mutant (without PYL2) and the PYL5::12458 complementation line remained fully open. b. Amplitudes of the changes in stomatal pore width from fully open to maximum closed after ABA infusion revealed that the presence of PYL2 is sufficient to close stomata to wild-type levels. Stomata of the 12458 mutant remained open. In contrast to all other samples, the width of PYL5::12458 stomata showed little but ABA-independent closure over time. c, Stomatal width of wild type and mutants after 20 min illumination. Only 12458 mutants showed substantially wider stomatal pores before ABA application. $n=9$, all replicate numbers represent biologically independent samples, mean \pm s.e.m.

there were also marked differences, and this must reflect the specific effects of these signals on guard cell properties. However, markedly, the integrated network analysis (see Supplementary Information 3 ) revealed that all closure-inducing signals induced changes in the expression of the $P Y R / P Y L / R C A R$ ABA receptor family. This provides a mechanism for all stimuli to modify stomatal function, 

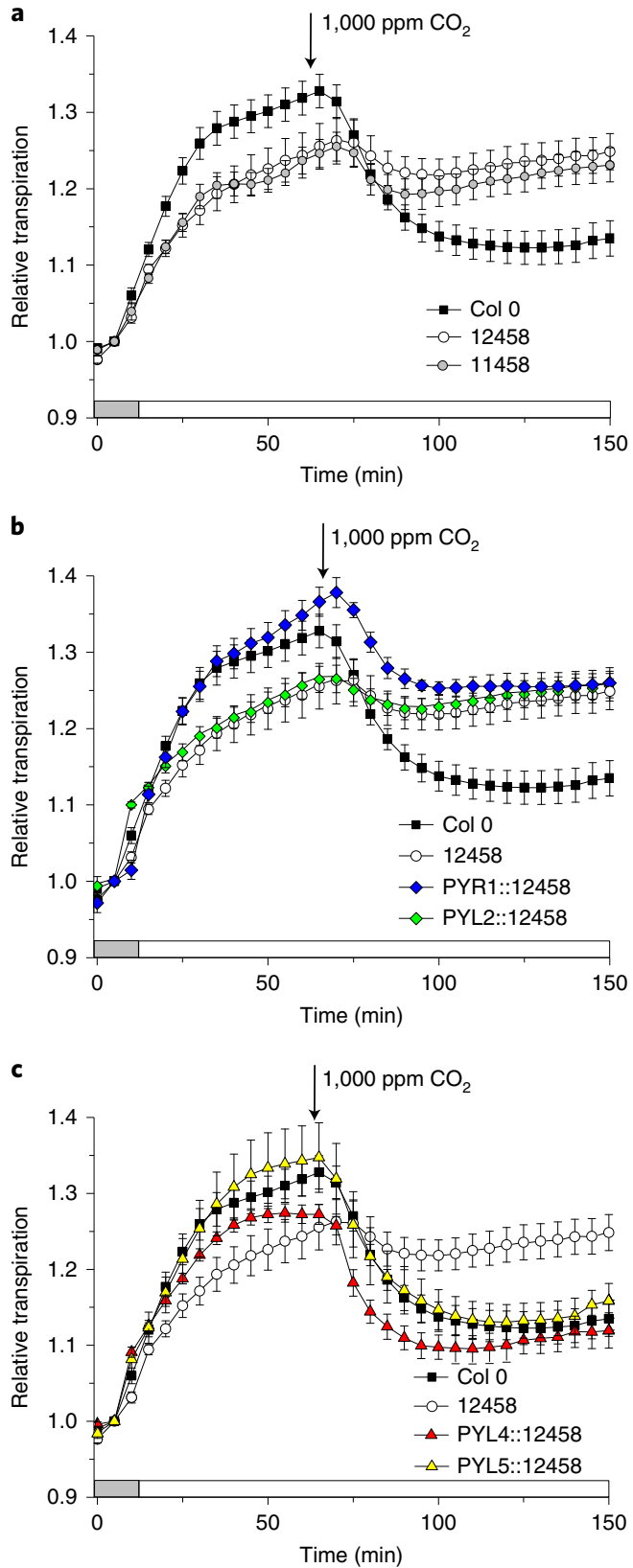

Fig. 5 | PYL4 and PYL5 render guard cells $\mathrm{CO}_{2}$ sensitive. Whole plants were placed in gas exchange cuvettes in the dark at $400 \mathrm{ppm} \mathrm{CO}_{2}$ and stomatal aperture was measured as a function of the relative transpiration, by gas exchange. Following stomatal opening in the light, the $\mathrm{CO}_{2}$ concentration was increased to $1,000 \mathrm{ppm}$. a, Stomata of wild-type plants closed about $60 \%$, while both quintuple mutants did not react. $\mathbf{b}$, The PYL2-complemented 12458 mutant did not react to elevated $\mathrm{CO}_{2}$ and the PYR1-complemented stomata also remained impaired. c, 12458 mutants complemented with either PYL4 or PYL5 closed their stomata in a wildtype manner. Values are normalized to time point $0 . n=20$ for $\mathrm{Col} 0, n=12$ for $12458, n=6$ for $11458, n=4$ for PYR1::12458, $n=6$ for PYL2::12458 and $n=7$ for PYL5::12458. All replicate numbers represent biologically independent samples, mean \pm s.e.m.

through modulating the sensitivity of the ABA-signalling pathway. We investigated the control of guard cell gene expression by ABA in more detail and found that individual genes, including members of the ABA receptor family, responded to ABA in a concentrationdependent manner. This conclusion was strengthened when we found that the expression of PYL4 or PYL5 in guard cells was sufficient for $\mathrm{CO}_{2}$-induced stomatal closure, but was not sufficient to restore the ability of ABA to inhibit light-induced stomatal opening in the 12458 mutant, whereas PYL2 was sufficient for this response. Our data on the ABA response of the complemented lines agree with a previous publication ${ }^{42}$, which showed that pyr1/pyl1/pyl2/ pyl4 quadruple mutant plants did not close their stomata following $\mathrm{ABA}$ application, and that $\mathrm{ABA}$ preincubation could not prevent stomatal opening by light. In contrast, in another study ${ }^{43}$ using the same mutant, ABA application to open stomata did not induce stomatal closure, but ABA preincubation prevented stomatal opening. In the latter paper the authors used guard cells in epidermal peels (obtained by macerating leaves in a Waring blender) floating on ABA solutions. Future studies will have to investigate whether, and how, mechanical stress feeds back onto the ABA biology of guard cells. It should be noted that the pyl2-1 single mutant ${ }^{12}$ behaved like the PYR1-complemented 12458 plants, indicating that PYR1 and PYL2 are the most important receptors for the fast ABA response of guard cells. However, one might not be able to exclude the possibility that either PYL8 or heterodimers involving receptors other than PYL2 are also competent to rescue the ABA-insensitive phenotype of the 12458 mutant.

In terms of stomatal $\mathrm{CO}_{2}$ responsiveness, a previous report agrees that $\mathrm{CO}_{2}$-induced stomatal closure is disrupted in $\mathrm{ABA}$ receptor mutant backgrounds ${ }^{8}$. In contrast, another paper concludes that 'rapid $\mathrm{CO}_{2}$ signal transduction leading to stomatal closure via an ABA-independent pathway' occurs ${ }^{44}$. How might these radically different conclusions be reconciled? It is, of course, only possible to speculate. However, as documented earlier ${ }^{23}$ stomatal responsiveness to ABA varies from insensitivity to sensitivity, and this is regulated by relative air humidity. Modulation of stomatal sensitivity by plant growth conditions, such as relative humidity, could provide a framework to explain the disparity in the results. The veracity of such a possibility requires further experimentation. Nevertheless, it is worth pointing out that examination of the data in Hsu et al. ${ }^{44}$, as in Merilo et al. ${ }^{7}$, reveals that the quadruple receptor mutants pyr1/pyl4/pyl5/pyls and hextuple pyr1/pyl1/pyl2/pyl4/pyl5/pyls display reduced $\mathrm{CO}_{2}$ responsiveness compared with wild type. These data do indicate a role for ABA receptors in the response to $\mathrm{CO}_{2}$. This was also the conclusion reached when stomatal conductance in response to $\mathrm{CO}_{2}$ was analysed in wild type and pyr1/pyl1/pyl2/ pyl4/pyl5/pyl8 ( ref. $^{7}$ ). Finally, the long-term exposures of the in-gel kinase assays done by Hsu et al. ${ }^{44}$ showed a basal activity of OST1 in guard cells, which, according to the authors, is probably necessary for rapid $\mathrm{CO}_{2}$-induced stomatal closure. Such a basal level of phosphorylation would be very sensitive to phosphatase and PYR/ PYL/RCAR activity, which can be modified by nanomolar changes in ABA concentration ( $K_{\mathrm{d}}$ for ternary complexes in the $20-40 \mathrm{nM}$ range $\left.^{11,13}\right)$. However, to return to the current investigation, our data, including its in-depth analysis of receptor mutants, are strongly supportive of a role for ABA receptors, in particular PYL4 and PYL5, in the stomatal response to increased concentrations of $\mathrm{CO}_{2}$.

Taken together our investigations reveal not only the complexity of the underlying cellular mechanisms responsible for controlling stomatal responses to closure-inducing signals, and how this might contribute towards stomatal response acclimation, but also a plausible mechanism for how stomata might integrate multiple signals. The results here are likely to be of benefit to plant breeders who are interested in engineering crops to respond to the complex multiple environmental stresses that are likely to be associated with future climates.

\section{Methods}

Plant material and growth conditions. Arabidopsis thaliana plants were grown in soil that was semi-sterilized for $20 \mathrm{~min}$ at $100^{\circ} \mathrm{C}$ and cultivated in climate chambers (Binder KBWF 720; www.binder-world.com) in a 12 -h day-night 
rhythm $\left(22 / 16^{\circ} \mathrm{C}, 60 \% \mathrm{RH}\right)$ and were illuminated with $125 \mu \mathrm{mol} \mathrm{m}^{-2} \mathrm{~s}^{-1}$ white light The gas exchange measurements and the expression studies were performed with 6-8-week-old, adult, non-flowering plants.

Preparation of the ABA receptor mutants. Using column genomic DNA as template we amplified the promoter region of Atlg22690 located 1,716 base pairs (bp) upstream of the start codon, named GC1 (ref. ${ }^{37}$ ), by PCR, using the primers GC1 forward 5'-ACCATGGAGTAAAGATTCAGTAACCC and GC1 reverse 5'-ATCCATGGTATTTCTTGAGTAGTGATTTTGAAG. Next, GC1 was cloned in pCR8/GW, excised as a NcoI fragment and cloned in front of either PYR1, PYL2, PYL4, PYL5 or PYL8. The resulting pGC1-PYR/PYL construct was recombined by LR reaction into modified pAlligator2 (ref. ${ }^{45}$ ), which has been previously doubly digested with HindIII-EcoRV, treated with Klenow fragment and religated to obtain a construct lacking the $35 \mathrm{~S}$ promoter. Each modified pAlligator2-GC1:PYR/PYL construct was transferred to Agrobacterium tumefaciens $\mathrm{C} 58 \mathrm{C} 1$ (pGV2260) ${ }^{46}$ by electroporation and used to transform 12458 and 11458 mutant plants ${ }^{28,33}$ by the floral dip method ${ }^{47}$. T1 transgenic seeds were selected on the basis of seed GFP fluorescence and sown in soil to obtain the T2 generation. Homozygous T3 progeny were used for further studies. Several PYL8:: 12458 complementation lines were also tested, but did not grow properly. They showed a severe phenotype and excised leaves wilted immediately. These mutants could not be used in any of our physiological experiments and were thus excluded from our analyses.

IRGA. Stomatal closing assays. Application of ABA and low air humidity were performed as described ${ }^{6}$. In brief, ABA solution $(50 \mu \mathrm{M}( \pm)$-ABA) was sprayed onto the plants until the surface was fully covered. The low-humidity treatment was performed by reducing the RH from $80 \%$ to $20 \%$ at $22^{\circ} \mathrm{C}$, which equals a VPD change from 0.529 to $2.116 \mathrm{kPa}$. High $\mathrm{CO}_{2}(1,000 \mathrm{ppm})$ was applied to plants in airtight boxes after $2 \mathrm{~h}$ illumination at ambient air to close stomata. For the samples in darkness, plants were fully shaded following $2 \mathrm{~h}$ of illumination. Each closing signal persisted for $4 \mathrm{~h}$. To minimize diurnal effects on gene expression, all procedures were started in the morning $2 \mathrm{~h}$ after onset of illumination.

Leaf gas exchange measurements. Transpiration rates were measured with a custom made system as described elsewhere ${ }^{38}$. Carbon dioxide response measurements were performed with intact plants at $20^{\circ} \mathrm{C}$ and $52.5 \pm 3 \% \mathrm{RH}(\mathrm{VPD}=1.123 \mathrm{~Pa})$ The soil surface of the pots was tightly covered with plastic foil to avoid water evaporation. After the transpiration rates had stabilized in the dark, plants were illuminated with $125 \mu \mathrm{mol} \mathrm{m}{ }^{-2} \mathrm{~s}^{-1}$ white light. After $1 \mathrm{~h}$ of illumination the $\mathrm{CO}_{2}$ concentration of the air stream was increased from $400 \mathrm{ppm}$ to $1,000 \mathrm{ppm}$.

The effect of ABA on the light-induced transpiration of Arabidopsis was measured with detached leaves and the same conditions as described for the $\mathrm{CO}_{2}$ measurements. To avoid xylem embolism, the leaves were cut under water. The petioles were directly transferred into $50 \mu \mathrm{M} \pm \mathrm{ABA}$ solution and incubated in darkness. After $1 \mathrm{~h} \mathrm{ABA}$ incubation leaves were illuminated with $125 \mu \mathrm{mol} \mathrm{m}^{-2} \mathrm{~s}^{-1}$ white light and the transpirational water loss was recorded.

ABA electro-infusion. This method was performed according to ref. ${ }^{48}$. Excised leaves from 5-6-week-old plants were attached to a Petri dish using double-sided tape and submerged in a bath solution $\left(1 \mathrm{mM} \mathrm{KCl}, 1 \mathrm{mM} \mathrm{CaCl}_{2}, 5 \mathrm{mM} \mathrm{MES/BTP,}\right.$ $\mathrm{pH}$ 6). The Petri dish was mounted to an upright microscope (Axioskop 2FS, Zeiss) and the leaves were left for incubation while being exposed to $125 \mu \mathrm{mol} \mathrm{m}{ }^{-2} \mathrm{~s}^{-1}$ light. Stomata on the abaxial side of the leaves were visualized with a water immersion objective (Achroplan $\mathrm{x} 63 / 0.9 \mathrm{~W}$, Zeiss) and images were recorded with a camera (CoolSNAP HQ, Visitron Systems) in 30-s intervals using VisiView (Visitron Systems) imaging software. Electrodes from borosilicate capillaries (inner/outer diameter $=0.56 / 1.0 \mathrm{~mm}$; Hilgenberg) were pulled on a horizontal laser puller (P2000, Sutter Instrument) to achieve resistances ranging from 60 to $100 \mathrm{M} \Omega$. The electrodes were tip-loaded with $50 \mu \mathrm{M}( \pm)$-ABA, backfilled with $300 \mathrm{mM} \mathrm{KCl}$ and connected by Ag/Ag half-cells to the head stage (HS-2A $\times 0.01$; Axon Instruments, Molecular Devices) of a TEVC amplifier (GeneClamp 500; Axon Instruments). Reference electrodes were prepared using capillaries filled with $300 \mathrm{mM} \mathrm{KCl}$, sealed with an agar plug $(2 \% \mathrm{w} / \mathrm{v}$ agarose, $300 \mathrm{mM} \mathrm{KCl}$ and connected to ground with an Ag/Ag half-cell). Microelectrodes were driven with a piezoelectric micromanipulator (MM3A; Kleindiek). Before electro-infusion the electrode was brought into close proximity with an open stoma and left for $10 \mathrm{~min}$ to ensure that no mechanical closing stimulus was applied. ABA or control solution was released from the capillary by a negative, 10-min, 1-nA current pulse. After an additional $10 \mathrm{~min}$ the image recording was stopped.

ABA spray experiments. Arabidopsis plants were treated with ABA via spraying until the leaves were moist. To investigate the dose dependency of the ABA receptor expression in wild-type plants, ABA solutions with 0 (control plants), $0.025,0.1,0.25,2.5$ and $25 \mu \mathrm{M}$ ABA in deionized water containing 1:10,000 Triton X-100 were used. To investigate ABA-regulated guard cell expression patterns, plants were sprayed with $25 \mu \mathrm{M}$ ABA solution or deionized water with Triton X-100. After $4 \mathrm{~h}$ of incubation, guard cells were sampled and RNA was extracted.
$\mathrm{CO}_{2}$ treatment for microarrays. Single, potted 6-7-week-old Arabidopsis thaliana $(\mathrm{Col} 0)$ plants were subjected to an air stream containing ambient $\mathrm{CO}_{2}$ $(380 \mathrm{ppm})$ and $50 \pm 5 \% \mathrm{RH}(\mathrm{VPD}=1.322 \mathrm{kPa})$ in climate chambers at $22 \pm 2{ }^{\circ} \mathrm{C}$ and $100 \mu \mathrm{mol} \mathrm{m}^{-2} \mathrm{~s}^{-1}$ white light. After $1 \mathrm{~h}$ half of the plants were treated with $1,000 \mathrm{ppm}$ $\mathrm{CO}_{2}$ for $4 \mathrm{~h}$. Guard cells were sampled and RNA was extracted for microarray hybridization.

Darkness treatment for microarrays. Single potted 6-7-week-old Arabidopsis thaliana (Col 0) plants were subjected to an air stream containing ambient $\mathrm{CO}_{2}$ $(400 \mathrm{ppm})$ and $50 \pm 5 \% \mathrm{RH}(\mathrm{VPD}=1.322 \mathrm{kPa})$ in climate chambers at $22 \pm 2{ }^{\circ} \mathrm{C}$ and $100 \mu \mathrm{mol} \mathrm{m}^{-2} \mathrm{~s}^{-1}$ white light. After $2 \mathrm{~h}$ half of the plants were kept in darkness for $2 \mathrm{~h}$. Guard cells were sampled and RNA was extracted for microarray hybridization.

ABA and low RH (increased VPD) treatment. These microarray data come from a previous study ${ }^{6}$ in which the ABA and low-humidity (increased VPD) signals persisted over $4 \mathrm{~h}$.

Sampling and RNA extraction. Guard cell sampling and RNA extraction were performed as described ${ }^{6}$. Guard cell samples were frequently tested via vital staining to confirm that contamination by other cell types remained below $5-10 \%$.

Quantitative real-time PCR. Quantitative real-time PCR ( $\mathrm{qPCR}$ ) was performed as described ${ }^{49}$. All transcripts were normalized to 10,000 molecules of actin2/8 and thus denoted as relative expression. All indicated replicate numbers refer to biological replicates. Primer sequences used: PYR1 fwd 5'-GCTGACGAATTACA AATCCGTT-3', PYR1 rev 5' -ACCGTCGCGAGTTTCTG-3', PYL1 fwd 5' -CGTG AACGTGATAAGTGG-3', PYL1 rev 5'-TGAACCGTCGTAACCGAT-3', PYL2 fwd 5'-CATAACCCAACGCATCCA-3' ${ }^{\prime}$, PYL 2 rev 5' -AACTCAAGCCGCTCGGTA-3', PYL4 fwd 5' -CCGCTCGTTTTCACACACAC-3', PYL2 rev 5'-GTGTTGCCTGG AGGAACATC-3', PYL5 fwd 5' -TGGTGCAGATGATCCACG-3', PYL4 rev 5' -AG ACTGAAGGTTGCACCG-3', PYL8 fwd 5' -TGTGTGGTCACTTGTGAG-3', PYL8 rev 5'-TGAACCGCAAGACGTTCA-3', KCR2 fwd 5'-ATGTGGATGCACTATC A-3', KCR2 rev $5^{\prime}$-AAGGTTATCCGGTACAA-3', ABI2 fwd $5^{\prime}$-GGACTTAGAG GCTATTG-3', ABI2 rev 5' -AGGATTAAATCCATTAGTG-3', MYB60 fwd 5' -ATG CTGTGACAAGATAGG-3', MYB60 rev 5' -AAAGTTTCCACGTTTAAT-3' CIPK25 fwd 5' -AGATCCAAAACGTAGAAG-3', CIPK25 rev 5'-CTTACACAACTCAAC GAC-3', HAI1 fwd 5'-GTTGAATAGTTTTGACGA-3', HAI1 rev 5'-GCCGTATT TAGGATAAGC-3', ABR fwd 5' -GGTGGAATGATGGACAAG-3', ABR rev 5'-AT AAAGATCCAAATGGACG-3', RAB18 fwd 5' -AGAAGGGAATAACACAAA-3', RAB18 rev 5' -CAATACAACGACCGAA-3'.

PYL2 protein identification. For the identification and quantification of PYL2, parallel reaction monitoring (PRM) via mass spectrometry was used. Four or five biological replicates from each experimental group were examined. Proteins were extracted from the ground guard cells following the phenol extraction/ammonium acetate precipitation method described ${ }^{50}$. Briefly, $150 \mathrm{mg}$ starting material was processed. For tryptic digestion, an aliquot of $20 \mu \mathrm{g}$ protein was digested with $0.2 \mu \mathrm{g}$ trypsin. Samples were cleaned using C18 solid phase extraction according to the manufacturer (Pierce C18 spin columns, ThermoFisher Scientific) and dissolved in $5 \%$ acetonitrile (ACN) and $0.1 \%$ formic acid (FA). Peptides from the candidate proteins were designed using Skyline (v.4.2) and ordered from ThermoFisher Scientific (PEPotec Grade 1). The ultra performance liquid chromatographytandem mass spectrometry (UPLC-MS/MS) analysis was performed on an Ultimate 3000 UPLC system (Dionex, Thermo Scientific) equipped with a C18 PepMap100 precolumn $(5 \mu \mathrm{m}, 300 \mu \mathrm{m} \times 5 \mathrm{~mm}$. Thermo Scientific) and an EasySpray C18 column $(3 \mu \mathrm{m}, 75 \mu \mathrm{m} \times 15 \mathrm{~cm}$, Thermo Scientific) using a gradient of $5 \%$ to $20 \%$ $\mathrm{ACN}$ in $0.1 \% \mathrm{FA}$ for $10 \mathrm{~min}$ followed by a gradient of $10 \%$ to $35 \% \mathrm{ACN}$ in $0.1 \% \mathrm{FA}$ for $4 \mathrm{~min}$ and a final gradient from $35 \%$ to $95 \% \mathrm{ACN}$ in $0.1 \% \mathrm{FA}$ for $2.5 \mathrm{~min}$ and a Q Exactive Orbitrap mass spectrometer (Thermo Scientific). The flow-rate was set at $250 \mu \mathrm{min}^{-1}$. The $\mathrm{Q}$ Exactive was operated in positive-ion mode with a nanospray voltage of $1.5 \mathrm{kV}$ and a source temperature of $250^{\circ} \mathrm{C}$. ProteoMass LTQ/FT-Hybrid ESI Positive Mode CalMix (MSCAL5-1EA SUPELCO, Sigma-Aldrich) was used as an external calibrant and the lock mass 445.12003 as an internal calibrant. For the characterization of the standard peptide library, the instrument was operated in a data-dependent acquisition mode with a survey MS scan at a resolution of 70,000 (full width at half maximum at $m / z 200$ ) for the mass range of $m / z 350-1,800$ for precursor ions, followed by MS/MS scans of the top ten most intense peaks with +2 , +3 and +4 charged ions above a threshold ion count of 16,000 at a 35,000 resolution using a normalized collision energy of $28 \mathrm{eV}$ with an isolation window of $3.0 \mathrm{~m} / \mathrm{z}$ and dynamic exclusion of $10 \mathrm{~s}$. All data were acquired with Xcalibur v.2.2 software (Thermo Scientific). The most suitable peptide per protein was chosen to set the PRM analysis. To avoid overlapping time windows the samples were analysed on the Q Exactive in PRM mode in two separate runs (see Table 3).

PRM analysis was performed in Skyline v.4.2. The dicyano-1,4-benzoquinone analysis of the peptide library was done with proteome discoverer and the library was loaded in Skyline as an msf file. The NCBI Arabidopsis fata file was loaded and the results of the PRM run were loaded as raw files with the following settings: MS1 orbitrap detection 70,000 resolution, MS2 orbitrap detection 20,000 resolution. Only peptides showing three accurate transitions were accepted. 


\begin{tabular}{|c|c|c|c|c|c|c|c|}
\hline $\begin{array}{l}\text { Peptide } \\
\text { name }\end{array}$ & Sequence & $\begin{array}{l}\text { Peptide } \\
\text { length }\end{array}$ & $m / z$ & $Z$ & Start & End & Run \\
\hline PYL2 & LISGDGDVGSVR & 12 & 587.8068 & 2 & 25.9 & 28.9 & 1 \\
\hline
\end{tabular}

'Start' and 'End' refer to the start and end times that the quadrupole window was open for the specified peptide, respectively.

Microarray hybridization. For transcriptome profiling, samples were hybridized on an Agilent Platform using the Agilent Arabidopsis v4 microarray chips (catalogue no. 021169) (http://www.agilent.com). RNA quantity was measured with an ND-100 Spectrophotometer v.3.3.0 (NanoDrop Technologies). RNA integrity was confirmed using an Agilent RNA 6000 Nano Chip on an Agilent 2100 BioAnalyzer (vB.02.03 BSI307). Total RNA (500 ng) was used for each sample labelling. Labelling and preparation of samples for hybridization was performed as described in the onecolour microarray-based gene-expression analysis protocol provided by Agilent, including the one-colour RNA spike-in kit (v.5.0.1, 2006; Agilent Technologies). Slides were scanned on the Agilent Microarray Scanner with extended dynamic range at high resolution $(5 \mu \mathrm{m})$. Datasets were extracted by propriety software package (v.9.5.3.1, Agilent Technologies) using a standard protocol.

Array analysis. Data preprocessing was performed using Bioconductor software ${ }^{5}$ with the statistical programming environment $\mathrm{R}^{52}$. Normalization has been performed using negative control probes and quantile normalization using negative and positive control probes as implemented in the neqc function ${ }^{53}$ of the limma package $^{54}$. Differential gene expression for all stimuli was calculated using the moderated $t$-statistic approach as implemented in the $\mathrm{R}$ package limma ${ }^{54}$, which has been specifically developed for the analysis of small sample size experiments. The $P$ values of all results were corrected for multiple testing by using the false discovery rate $(\mathrm{FDR})^{55}$. To identify guard cell-specific gene regulation, we used a meta-analysis approach based on the order statistic of the stimulus $P$ values. Therefore, $P$ values from a one-sided moderated $t$-test of differentially expressed genes between guard cells and leaf tissue have been derived from the limma model. Subsequently, for each stimulus a combined $P$ value has been calculated using the second-order statistics of the stimulus and the guard cell versus leaf $P$ value. This results in a set of genewise $P$ values for guard cell-specific stimulus effects.

Pathway enrichment analyses. Analysis was carried out by a model-based approach, which can be integrated directly into the limma analysis of differential expression. A competitive gene-set test has been applied to all stimulation contrasts, as implemented in the function camera ${ }^{56}$. This function tests whether a set of genes is highly ranked relative to other genes, in terms of differential expression, accounting for potential intergene correlation. Pathways for the analysis have been obtained from the MapMan catalogue ${ }^{57}$ based on TAIR v. 10 (2012), from which all gene sets with more than 5 genes and fewer than 500 genes have been included in the analysis.

Integrative network analysis. Network data has been obtained from the STRING database (v.9.0, http://string-db.org) $)^{58}$. All interactions of the genome of Arabidopsis thaliana have been extracted from the database, yielding a total of 717,810 interactions between 16,465 genes. For the analysis we used the high confidence network (combined score $>0.7$ ) resulting in 69,603 interactions between 7,090 genes. After mapping all genes on the array to the network, the resulting largest connected component comprising 69,329 interactions between 6,724 genes has been extracted. This constitutes the base network used in all subsequent network analyses.

An optimal algorithm has been established to decompose the large intricate network structure into functionally coherent network modules ${ }^{59}$. These modules can be viewed as building blocks of cellular processes, such as basic metabolic pathways or stimuli-specific signalling. To identify these signalling subnetworks the genes (network nodes) need to be scored according to their measured regulation, and responsive modules can be identified using an exact search algorithm.

Here, a statistical model to derive functional scores of closing signal responsiveness in guard cells has been used. Therefore, all stimuli $P$ values have been derived from the limma analysis as detailed above. Integrated network analysis node (gene) scores have been computed based on these $P$ values as detailed in ref. ${ }^{59}$ using the routines implemented in the R package BioNet ${ }^{60}$ Briefly, a beta-uniform mixture model has been fitted to the $P$ value distribution, thereby decomposing the signal and noise components of the distribution. Based on this, network scores have been calculated as the log likelihood ratio of the signal to the noise component. Thus, positive scores reflect signal content (low $P$ values), whereas the negative scores reflect non-significant genes (noise). The signal to noise threshold has been adjusted by multiple testing using the FDR. For the different stimuli, appropriate FDR values have been selected according to the different effect strengths controlling the size of the resulting modules. These mainly contain genes with a maximal response, but also a few tightly associated genes that show no differential regulation on the transcriptomic level (represented as squares in the network). Subsequently, an exhaustive network search has been performed on the entire node-scored network to identify the maximum-scoring subnetworks using an exact algorithm ${ }^{59}$. The resulting optimal solutions represent maximally significant, differentially regulated modules. On the basis of functional scoring of stimulation, this constitutes the optimal responsive modules within the entire network. This also means that no other module shows a stronger guard cellspecific closing signal response.

Statistics. To test significances on qPCR (Tables 1 and 2 and Supplementary Figs. 3 and 6) we used a multivariable linear model on log-transformed expression values (relative to actin), adjusting additionally for the day of experiment. Heteroscedasticity-consistent standard errors for regression coefficients have been calculated as implemented in the sandwich package using the default setting ${ }^{61}$. For dose-response experiments (Supplementary Fig. 3) comparisons of different concentrations to the unstimulated control have been multiple-testing corrected on the basis of Dunnet's post hoc test procedure for many-to-one comparisons. We used the step-down adjustment (method = 'free') as implemented in the 'multcomp' package in $\mathrm{R}^{62}$. Reported $P$ values are based on two-sided tests, $P$ values $<0.05$ are regarded as significant. Levels of significance were ${ }^{\star} P \leq 0.05,{ }^{*} P \leq 0.01,{ }^{* *} P \leq 0.001$.

Reporting Summary. Further information on research design is available in the Nature Research Reporting Summary linked to this article.

\section{Data availability}

The data that support the findings of this study are available from the corresponding authors upon reasonable request. Microarray data of the ABA and low air humidity treatments that were taken from ref. ${ }^{6}$ were deposited in the Gene Expression Omnibus (GEO) database http://www.ncbi.nlm.nih.gov/geo with accession no. GSE41054. The microarray data from $\mathrm{CO}_{2}$ and darkness experiments were deposited in the same database under GSE1 18520.

\section{Code availability}

Algorithms and statistics used in the analyses are based on published approaches available in R packages (mainly Bioconductor framework) and other cited publicly available repositories.

Received: 6 August 2018; Accepted: 9 July 2019; Published online: 26 August 2019

\section{References}

1. Hetherington, A. M. \& Woodward, F. I. The role of stomata in sensing and driving environmental change. Nature 424, 901-908 (2003).

2. Vialet-Chabrand, S. R. M. et al. Temporal dynamics of stomatal behavior: modeling and implications for photosynthesis and water use. Plant Physiol. 174, 603-613 (2017)

3. Lawson, T. \& Blatt, M. R. Stomatal size, speed, and responsiveness impact on photosynthesis and water use efficiency. Plant Physiol. 164, 1556-1570 (2014).

4. Assmann, S. M. \& Jegla, T. Guard cell sensory systems: recent insights on stomatal responses to light, abscisic acid, and $\mathrm{CO}_{2}$. Curr. Opin. Plant Biol. 33, 157-167 (2016)

5. Engineer, $\mathrm{C}$. B. et al. $\mathrm{CO}_{2}$ sensing and $\mathrm{CO}_{2}$ peculation of stomatal conductance: advances and open questions. Trends Plant Sci. 21, 16-30 (2016).

6. Bauer, $\mathrm{H}$. et al. The stomatal response to reduced relative humidity requires guard cell-autonomous ABA synthesis. Curr. Biol. 23, 53-57 (2013).

7. Merilo, E. et al. PYR/RCAR receptors contribute to ozone-, reduced air humidity-, darkness- and $\mathrm{CO}_{2}$-induced stomatal regulation. Plant Physiol. 162, 1652-1668 (2013).

8. Chater, $\mathrm{C}$. et al. Elevated $\mathrm{CO}_{2}$-induced responses in stomata require $\mathrm{ABA}$ and ABA signaling. Curr. Biol. 25, 2709-2716 (2015).

9. Hedrich, R. \& Geiger, D. Biology of SLAC1-type anion channels - from nutrient uptake to stomatal closure. New Phytol. 216, 46-61 (2017).

10. Scherzer, S., Maierhofer, T., Al-Rasheid, K. A. S., Geiger, D. \& Hedrich, R. Multiple calcium-dependent kinases modulate ABA-activated guard cell anion channels. Mol. Plant 5, 1409-1412 (2012).

11. Ma, Y. et al. Regulators of PP2C phosphatase activity function as abscisic acid sensors. Science 324, 1064-1068 (2009).

12. Park, S. Y. et al. Abscisic acid inhibits type $2 \mathrm{C}$ protein phosphatases via the PYR/PYL family of START proteins. Science 324, 1068-1071 (2009).

13. Santiago, J. et al. Modulation of drought resistance by the abscisic acid receptor PYL5 through inhibition of clade A PP2Cs. Plant J. 60, 575-588 (2009).

14. Munemasa, S. et al. Mechanisms of abscisic acid-mediated control of stomatal aperture. Curr. Opin. Plant Biol. 28, 154-162 (2015).

15. Hedrich, R. Ion channels in plants. Physiol. Rev. 92, 1777-1811 (2012).

16. Franks, P. J., Leitch, I. L., Ruszala, E. M., Hetherington, A. M. \& Beerling, D. J. Physiological framework for adaptation of stomata to $\mathrm{CO}_{2}$ from glacial to future concentrations. Philos. Trans. R. Soc. B 367, 537-546 (2012). 
17. Woodward, F. I. Stomatal numbers are sensitive to increases in $\mathrm{CO}_{2}$ from preindustrial levels. Nature 327, 617-618 (1987).

18. Gray, J. E. et al. The HIC signalling pathway links $\mathrm{CO}_{2}$ perception to stomatal development. Nature 408, 713-716 (2000).

19. Doheny-Adams, T., Hunt, L., Franks, P. J., Beerling, D. J. \& Gray, J. E. Genetic manipulation of stomatal density influences stomatal size, plant growth and tolerance to restricted water supply across a growth carbon dioxide gradient. Philos. Trans. R. Soc. B 367, 547-555 (2012).

20. Casson, S. A. et al. Phytochrome B and PIF4 regulate stomatal development in response to light quantity. Curr. Biol. 19, 229-234 (2009).

21. Casson, S. A. \& Hetherington, A. M. Phytochrome B is required for light-mediated systemic control of stomatal development. Curr. Biol. 24, 1216-1221 (2014).

22. Franks, P. J., T, W. D.-A., Britton-Harper, Z. J. \& Gray, J. E. Increasing water-use efficiency directly through genetic manipulation of stomatal density. New Phytol. 207, 188-195 (2015).

23. Pantin, F. et al. Developmental priming of stomatal sensitivity to abscisic acid by leaf microclimate. Curr. Biol. 23, 1805-1811 (2013).

24. Aliniaeifard, S. \& van Meeteren, U. Can prolonged exposure to low VPD disturb the ABA signalling in stomatal guard cells? J. Exp. Bot. 64 3551-3566 (2013).

25. Obayashi, T., Nishida, K., Kasahara, K. \& Kinoshita, K. ATTED-II updates: condition-specific gene coexpression to extend coexpression analyses and applications to a broad range of flowering plants. Plant Cell Physiol. 52, 213-219 (2011).

26. Bauer, $H$. et al. How do stomata sense reductions in atmospheric relative humidity? Mol. Plant 6, 1703-1706 (2013).

27. Kong, W. et al. Two novel flavin-containing monooxygenases involved in biosynthesis of aliphatic glucosinolates. Front. Plant Sci. 7, 1292 (2016).

28. Gonzalez-Guzman, M. et al. Arabidopsis PYR/PYL/RCAR receptors play a major role in quantitative regulation of stomatal aperture and transcriptional response to abscisic acid. Plant Cell 24, 2483-2496 (2012).

29. Weng, J. K., Ye, M., Li, B. \& Noel, J. P. Co-evolution of hormone metabolism and signaling networks expands plant adaptive plasticity. Cell 166, 881-893 (2016)

30. Zhao, Y. et al. Arabidopsis duodecuple mutant of PYL ABA receptors reveals PYL repression of ABA-independent SnRK2 activity. Cell Rep. 23, 3340-3351 (2018).

31. Antoni, R. et al. Selective inhibition of clade A phosphatases type $2 \mathrm{C}$ by PYR/PYL/RCAR abscisic acid receptors. Plant Physiol. 158, 970-980 (2012).

32. Tischer, S. V. et al. Combinatorial interaction network of abscisic acid receptors and coreceptors from Arabidopsis thaliana. Proc. Natl Acad. Sci. USA 114, 10280-10285 (2017)

33. Antoni, R. et al. Pyrabactin resistancel-like 8 plays an important role for the regulation of abscisic acid signaling in root. Plant Physiol. 161 931-941 (2013).

34. Belda-Palazon, B. et al. PYL8 mediates ABA perception in the root through non-cell-autonomous and ligand-stabilization-based mechanisms. Proc. Natl Acad. Sci. USA 115, E11857-E11863 (2018)

35. Zhao, Y. et al. ABA receptor PYL9 promotes drought resistance and leaf senescence. Proc. Natl Acad. Sci. USA 113, 1949-1954 (2016).

36. Pri-Tal, O., Shaar-Moshe, L., Wiseglass, G., Peleg, Z. \& Mosquna, A. Non-redundant functions of the dimeric ABA receptor BdPYL1 in the grass Brachypodium. Plant J. 92, 774-786 (2017).

37. Yang, Y., Costa, A., Leonhardt, N., Siegel, R. S. \& Schroeder, J. I. Isolation of a strong Arabidopsis guard cell promoter and its potential as a research tool. Plant Methods 4, 6 (2008).

38. Muller, H. M. et al. The desert plant Phoenix dactylifera closes stomata via nitrate-regulated SLAC1 anion channel. New Phytol. 216, 150-162 (2017).

39. Ache, P. et al. Stomatal action directly feeds back on leaf turgor: new insights into the regulation of the plant water status from non-invasive pressure probe measurements. Plant J. 62, 1072-1082 (2010).

40. Raschke, K. Simultaneous requirement of carbon dioxide and abscisic acid for stomatal closing in Xanthium strumarium L. Planta 125, 243-259 (1975).

41. Webb, A. A. \& Hetherington, A. M. Convergence of the abscisic acid, $\mathrm{CO}_{2}$, and extracellular calcium signal transduction pathways in stomatal guard cells. Plant Physiol. 114, 1557-1560 (1997).

42. Nishimura, N. et al. PYR/PYL/RCAR family members are major in-vivo ABI1 protein phosphatase 2C-interacting proteins in Arabidopsis. Plant J. 61, 290-299 (2010).

43. Yin, Y. et al. Difference in abscisic acid perception mechanisms between closure induction and opening inhibition of stomata. Plant Physiol. 163 600-610 (2013).

44. Hsu, P. K. et al. Abscisic acid-independent stomatal $\mathrm{CO}_{2}$ signal transduction pathway and convergence of $\mathrm{CO}_{2}$ and ABA signaling downstream of OST1 kinase. Proc. Natl Acad. Sci. USA 115, E9971-E9980 (2018).

45. Bensmihen, S. et al. Analysis of an activated ABI5 allele using a new selection method for transgenic Arabidopsis seeds. FEBS Lett. 561, 127-131 (2004).
46. Deblaere, R. et al. Efficient octopine Ti plasmid-derived vectors for Agrobacterium-mediated gene transfer to plants. Nucleic Acids Res. 13, 4777-4788 (1985)

47. Clough, S. J. \& Bent, A. F. Floral dip: a simplified method for Agrobacteriummediated transformation of Arabidopsis thaliana. Plant J. 16, 735-743 (1998).

48. Huang, $\mathrm{S}$. et al. $\mathrm{Ca}^{2+}$ signals in guard cells enhance the efficiency by which ABA triggers stomatal closure. New Phytol. https://doi.org/10.1111/nph.15985 (2019).

49. Geiger, D. et al. Stomatal closure by fast abscisic acid signaling is mediated by the guard cell anion channel SLAH3 and the receptor RCAR1. Sci. Signal 4, ra32 (2011)

50. Geilfus, C. M., Tenhaken, R. \& Carpentier, S. C. Transient alkalinization of the leaf apoplast stiffens the cell wall during onset of chloride salinity in corn leaves. J. Biol. Chem. 292, 18800-18813 (2017).

51. Huber, W. et al. Orchestrating high-throughput genomic analysis with Bioconductor. Nat. Methods 12, 115-121 (2015).

52. R Development Core Team. R: A Language and Environment for Statistical Computing (R Foundation for Statistical Computing, 2011).

53. Shi, W., Oshlack, A. \& Smyth, G. K. Optimizing the noise versus bias trade-off for Illumina whole genome expression BeadChips. Nucleic Acids Res. 38, e204 (2010).

54. Ritchie, M. E. et al. limma powers differential expression analyses for RNA-sequencing and microarray studies. Nucleic Acids Res. 43, e47 (2015).

55. Benjamini, Y. \& Hochberg, Y. Controlling the false discovery rate a practical and powerful approach to multiple testing. J. R. Stat. Soc. B 57, 289-300 (1995).

56. Wu, D. \& Smyth, G. K. Camera: a competitive gene set test accounting for inter-gene correlation. Nucleic Acids Res. 40, e133 (2012).

57. Gentleman, R. C. et al. Bioconductor: open software development for computational biology and bioinformatics. Genome Biol. 5, R80 (2004).

58. Szklarczyk, D. et al. The STRING database in 2011: functional interaction networks of proteins, globally integrated and scored. Nucleic Acids Res. 39, D561-D568 (2011).

59. Dittrich, M. T., Klau, G. W., Rosenwald, A., Dandekar, T. \& Muller, T. Identifying functional modules in protein-protein interaction networks: an integrated exact approach. Bioinformatics 24, i223-i231 (2008).

60. Beisser, D., Klau, G. W., Dandekar, T., Muller, T. \& Dittrich, M. T. BioNet: an R-package for the functional analysis of biological networks. Bioinformatics 26, 1129-1130 (2010)

61. Zeileis, A. Econometric computing with HC and HAC covariance matrix estimators. J. Stat. Softw. 11, 1-17 (2004).

62. Hothorn, T., Bretz, F. \& Westfall, P. Simultaneous inference in general parametric models. Biom. J. 50, 346-363 (2008).

\section{Acknowledgements}

Work in the Hedrich laboratory was supported by a grant from King Saud University Deanship for Scientific Research, International Research Group Programme (IRG14-22), Riyadh, Saudi Arabia, and that of the Rodriguez laboratory was supported by the Ministerio de Ciencia e Innovacion, Fondo Europeo de Desarrollo Regional and Consejo Superior de Investigaciones Cientificas (grants BIO2014-52537-R and BIO2017-82503-R, to P.L.R.). M.D. was supported by the CRC/Transregio 124 - FungiNet funded by the Deutsche Forschungsgemeinschaft (project B2). A.M.H. acknowledges support from the UK BBSRC (grant no. BB/N001168/1). E.M. was supported by the Estonian Research Council (grant no. PUT1133).

\section{Author contributions}

M.D. and T.M. conceived and conducted bioinformatics. H.M.M., H.B. and P.A. conceived, performed and analysed the expression studies. H.M.M. and E.M. conducted and analysed gas exchange measurements. M.P.-L. and P.L.R. conceived and conducted the generation of transgenic plants. C.-M.G. and S.C.C. conceived and conducted proteomic analyses. J.H. conceived, conducted and analysed electro-infusion experiments. P.A., P.L.R., H.K., K.A.S.A.-R., T.M., A.M.H. and R.H. designed and conceived the study. M.D., T.M., P.A., A.M.H. and R.H. wrote the manuscript. All authors discussed the results and commented on the manuscript.

\section{Competing interests}

The authors declare no competing interests.

\section{Additional information}

Supplementary information is available for this paper at https://doi.org/10.1038/ s41477-019-0490-0.

Reprints and permissions information is available at www.nature.com/reprints. Correspondence and requests for materials should be addressed to T.M. or A.M.H Publisher's note: Springer Nature remains neutral with regard to jurisdictional claims in published maps and institutional affiliations.

(c) The Author(s), under exclusive licence to Springer Nature Limited 2019 


\section{Reporting Summary}

Nature Research wishes to improve the reproducibility of the work that we publish. This form provides structure for consistency and transparency in reporting. For further information on Nature Research policies, see Authors \& Referees and the Editorial Policy Checklist.

\section{Statistical parameters}

When statistical analyses are reported, confirm that the following items are present in the relevant location (e.g. figure legend, table legend, main text, or Methods section).

n/a $\mid$ Confirmed

$\square$ The exact sample size $(n)$ for each experimental group/condition, given as a discrete number and unit of measurement

$\square$ An indication of whether measurements were taken from distinct samples or whether the same sample was measured repeatedly

$\square$ The statistical test(s) used AND whether they are one- or two-sided

$\square$ Only common tests should be described solely by name; describe more complex techniques in the Methods section.

$\square$ A description of all covariates tested

$\square$ \A description of any assumptions or corrections, such as tests of normality and adjustment for multiple comparisons

$\square$ A full description of the statistics including central tendency (e.g. means) or other basic estimates (e.g. regression coefficient) AND

$\triangle$ variation (e.g. standard deviation) or associated estimates of uncertainty (e.g. confidence intervals)

$\square$ For null hypothesis testing, the test statistic (e.g. $F, t, r$ ) with confidence intervals, effect sizes, degrees of freedom and $P$ value noted

$\square$ Give $P$ values as exact values whenever suitable.

Х $\square$ For Bayesian analysis, information on the choice of priors and Markov chain Monte Carlo settings

\ $\square$ For hierarchical and complex designs, identification of the appropriate level for tests and full reporting of outcomes

$\bigotimes \square$ Estimates of effect sizes (e.g. Cohen's $d$, Pearson's $r$ ), indicating how they were calculated

$\varnothing$ Clearly defined error bars

State explicitly what error bars represent (e.g. SD, SE, CI)

Our web collection on statistics for biologists may be useful.

\section{Software and code}

Policy information about availability of computer code

Data collection We used for guard cell imaging VisiView v2.1.4 (Visitron Systems), for proteomics Xcalibur v2.2 (Thermo Fisher Scientific), network data have been obtained from the STRING database v9.0

Data analysis

We used Excel 2016-2018, for proteomics Skyline v4.2 (MacCoss Lab Software), for microarray analyses and the modules R v3.2.2 and the packages limma v3.26.9, BioNet v1.30.0, multcomp v1.4-6, and MapMan vTAIR10

For manuscripts utilizing custom algorithms or software that are central to the research but not yet described in published literature, software must be made available to editors/reviewers upon request. We strongly encourage code deposition in a community repository (e.g. GitHub). See the Nature Research guidelines for submitting code \& software for further information.

\section{Data}

Policy information about availability of data

All manuscripts must include a data availability statement. This statement should provide the following information, where applicable:

- Accession codes, unique identifiers, or web links for publicly available datasets

- A list of figures that have associated raw data

- A description of any restrictions on data availability

Microarray data were deposited in the Gene Expression Omnibus (GEO) database with accession no. GSE41054 for ABA and low air humidity treatments, and under 
The data that support the findings of this study are available from the corresponding

\section{Field-specific reporting}

Please select the best fit for your research. If you are not sure, read the appropriate sections before making your selection.
\ Life sciences
$\square$ Behavioural \& social sciences
Ecological, evolutionary \& environmental sciences

For a reference copy of the document with all sections, see nature.com/authors/policies/ReportingSummary-flat.pdf

\section{Life sciences study design}

All studies must disclose on these points even when the disclosure is negative.
Sample size
Sample size was not calculated but determined based on previous studies and experiences. Sample sizeof all experiments was sufficient to result in statistical significance and reproducibility. All replicates represent biologically independent samples. As is common practice, the design of the microarray analysis is based on four biological replicates for each condition.

Data exclusions

Replication

Density plots of raw signal intensities indicated two microarray outliers which have been removed prior subsequent analyses.

All experiments were conducted at least three times and gave similar results. For each type of reproduced experiment, plants grown under identical conditions, of the same age, and always at the same time of day were used to minimize batch effects.

Microarray data have been replicated and validated by qPCR as described in Bauer et al. (2013), Curr Biol, 23, 53-57 and Bauer et al. (2013), Mol Plant, 6, 1703-1706.

Randomization Plants for each experiment were allways chosen randomly

Blinding

\section{Reporting for specific materials, systems and methods}

Materials \& experimental systems

\begin{tabular}{l|l}
\hline $\mathrm{n} / \mathrm{a}$ & Involved in the study \\
\hline & $\square$ Unique biological materials \\
$\square$ & $\square$ Antibodies \\
$\square$ & $\square$ Animals and other organisms \\
\hline
\end{tabular}

\section{Unique biological materials}

Policy information about availability of materials

Obtaining unique materials All unique materials used in this study are readily availyble from the authors

\begin{tabular}{l|l}
\multicolumn{2}{l}{ Methods } \\
\hline n/a Involved in the study \\
$\square$ \\
$\square$ ChIP-seq \\
$\square$ \\
$\square$ Frow cytometry
\end{tabular}

